\title{
IMPLICIT MODELLING OF GEOLOGICAL STRUCTURES: A CARTESIAN GRID METHOD HANDLING DISCONTINUITIES WITH GHOST POINTS
}

\author{
JULIEN RENAUDEAU ${ }^{1,2}$, MODESTE IRAKARAMA ${ }^{2}$, GAUTIER LAURENT $^{2}$, \\ FRANTZ MAERTEN ${ }^{1} \&$ GUILLAUM CAUMON $^{2}$ \\ ${ }^{1}$ Schlumberger Petroleum Services, Montpellier, France \\ ${ }^{2}$ Université de Lorraine, CNRS, GeoRessources, Nancy, France
}

\begin{abstract}
In geology, implicit structural modelling constructs the geometry of geological structures (e.g. layers) by interpolating between sparse field data. A model is represented by a volumetric scalar field which is discontinuous on structural discontinuities such as faults or stratigraphic unconformities. The management of such discontinuities may involve boolean operations on several scalar fields or the creation of conformal meshes. Instead, we propose a ghost cell technique for the cartesian grid together with a set of relations between the ghost points, the regular nodes, and the discontinuities. Consequently, poor quality meshes are avoided and only the resolution of the grid has an impact on the solution. The modelling problem is posed as a least square's minimization of a bending energy penalization on data mismatch functions and approximated by finite difference. As all relationships in the grid are implicitly defined, except close to the discontinuities, this algorithm is computationally efficient. We provide some benchmarks of the method on two-dimensional examples with folds, faults, and erosions.
\end{abstract}

Keywords: 2D structural modelling, mesh reduction methods, energy penalization, ghost points.

\section{INTRODUCTION}

Implicit structural modelling consists in interpreting available field data into a model representing subsurface geological structures [1]-[8]. The model is represented by a scalar function defined on the entire volume of interest; this scalar function is called the implicit function. Horizons (interfaces between stratigraphic layers) are given by iso-values of the function, and structural discontinuities are given by discontinuous jumps in the function. Input data are represented by different types of modelling objects, such as points, vectors, polylines and surfaces [9]. The output model should represent natural geological structures while honouring the data.

The discrete smooth interpolation (DSI) [1], [4], [10] is an algorithm that discretises the implicit function on a background volumetric mesh where the mesh nodes are duplicated on either side of the discontinuities. The construction of such a mesh can represent a challenge depending on the geometrical complexity of the region being studied [11], [12]. The implicit function is then obtained by a spatial regression of data points sorted by horizons. DSI poses this problem as a least square's minimization of both this data regression and a roughness factor, obtaining a unique solution which should be as smooth as possible. In practice, this roughness factor has been designed to minimize the difference between the gradient of the implicit function evaluated on adjacent elements [4], [8], which is dependent on the mesh.

A version of DSI based on the Cartesian grid is introduced in [14]. The roughness factor is designed to minimize second directional derivatives of the implicit function using a finite difference approximation, and the implicit function is interpolated on the grid cells using a piecewise bilinear interpolation. The discontinuities are handled by deleting the grid cells they affect, putting those cells out of bounds of the model, which implies that the grid resolution is fine enough to include stratigraphic data in-bounds. The global grid resolution 


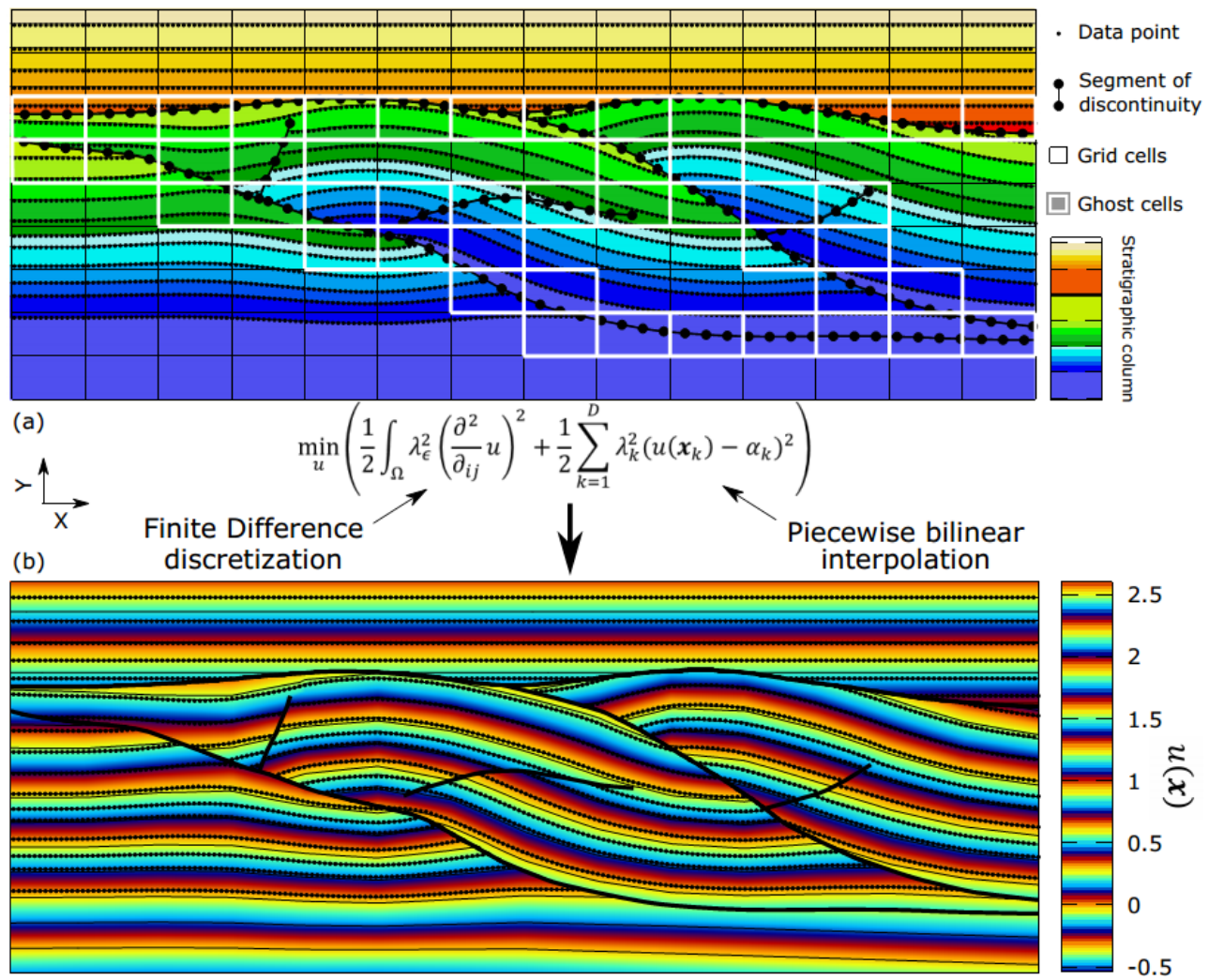

Figure 1: The proposed method applied on a synthetic cross section in two dimensions with folds, intersected faults and an erosion (figure modified from [13] with the present method). (a) The input data and the used interpolation grid; (b) The output structural model with extracted iso-values (fine lines).

is therefore determined based on the smallest distance of input data points to discontinuities, instead of being controlled by the complexity of the studied structures.

The roughness factor in DSI is defined as a discrete operator [10]. In practice, this factor may take different forms depending on the chosen domain discretisation scheme [4], [8], [14]. Instead, another approach is to find a continuous operator based on physical arguments, and then discretise it accordingly [13], [15]. An advantage of the latter is that it naturally provides appropriate weights to use during the least square's optimization stage, making the solution less sensitive to the resolution of the discretisation scheme.

In this paper, the proposed implicit method penalizes data constraints by the continuous bending energy, as in [13] and [15]. This problem is discretised on the Cartesian grid, using a piecewise bilinear evaluation on data terms and a finite difference approximation of energy terms, which gives a final linear system to solve close to [14], but with volumetric weights obtained from the discretisation. The discontinuities are handled with a ghost-cell technique, resulting in an implicit function defined everywhere in the domain of study. The proposed method has no requirement on the grid resolution other than to capture the studied geological structures. Fig. 1 summarizes the method on a synthetic cross section in two dimensions. 
The first section of this article presents the proposed modelling method, and the second gives additional practical information on the way ghost nodes are handled and the related limits.

\section{GRID BASED IMPLICIT STRUCTURAL MODELLING}

The implicit function $u(\boldsymbol{x})$ is defined in the vector space $V$ as

$$
V=\left\{\begin{array}{l|l}
u(\boldsymbol{x})=\sum_{l=1}^{N} \Phi_{l}(\boldsymbol{x}) u_{l}=\boldsymbol{\Phi}(\boldsymbol{x})^{T} \cdot \boldsymbol{U} & \boldsymbol{x}(x, y) \in \Omega
\end{array}\right\}
$$

where $\Omega \subset \mathbb{R}^{2}$ is the domain of study, $\boldsymbol{\Phi}^{T}=\left[\Phi_{1}, \ldots, \Phi_{N}\right]$ is a set of linearly independent functions forming the basis of functions, and $\boldsymbol{U}^{T}=\left[u_{1}, \ldots, u_{N}\right]$ is a set of constants.

Implicit structural modelling consists in finding the unknown coefficients $u_{l}$ to construct the implicit function $u(\boldsymbol{x})$ (eqn (1)) that represents geological strata while honouring the input data. To achieve this, the proposed method performs a spatial regression of data points with a bending energy penalization (or thin plate energy) [16], [17] by minimizing

$$
J(u)=\frac{1}{2} \int_{\Omega} \lambda_{\epsilon}^{2}\left(\left(\frac{\partial^{2}}{\partial_{x x}} u\right)^{2}+\left(\frac{\partial^{2}}{\partial_{y y}} u\right)^{2}+2\left(\frac{\partial^{2}}{\partial_{x y}} u\right)^{2}\right)+\frac{1}{2} \sum_{k=1}^{D} \lambda_{k}^{2}\left(u\left(\boldsymbol{x}_{k}\right)-\alpha_{k}\right)^{2},
$$

with $\lambda_{\epsilon}$ the energy penalization weight, $\lambda_{k}$ and $\alpha_{k}$ the weight and the expected value at a data point position $\boldsymbol{x}_{k}$, and $D$ the number of data points.

\subsection{Construction of the implicit function}

\subsubsection{Grid discretisation}

The domain $\Omega$ is discretised on a regular grid of $N$ nodes $\boldsymbol{x}_{l}$ (i.e., the grid corner points). Each coefficient $u_{l}$ is associated to a node $\boldsymbol{x}_{l}$, and the implicit function $u(\boldsymbol{x})$ is uniquely defined by these coefficients $u_{l}$ as the shape functions $\Phi_{l}(\boldsymbol{x})$ are linearly independent. Such a grid is illustrated in Fig. 1.

\subsubsection{Bilinear quadrilateral interpolation functions}

The implicit function coefficients $u_{l}$ are interpolated within the grid elements with bilinear functions $\Phi_{l}(\boldsymbol{x})$. These are created with the isoparametric quadrilateral elements with four nodes (i.e., the corners only) [18]. For any position $\boldsymbol{x}$ in $\Omega$, the containing cell $c$ is found. The position $\boldsymbol{x}(x, y)$ and the four cell nodes $\boldsymbol{x}_{l} \in c$ are transformed in the cell space as the position $\xi(\xi, \eta)$ and the four corners $\xi_{1}^{c}(-1,-1), \xi_{2}^{c}(1,-1), \xi_{3}^{c}(1,1)$ and $\xi_{4}^{c}(-1,1)$. The interpolation functions $N_{1}^{c}(\xi), N_{2}^{c}(\xi), N_{3}^{c}(\xi)$ and $N_{4}^{c}(\xi)$ in this space associated to the corresponding functions $\Phi_{l}(\boldsymbol{x})$ are defined as

$$
\begin{array}{ll}
N_{1}^{c}(\xi)=\frac{1}{4}(1-\xi)(1-\eta), & N_{3}^{c}(\xi)=\frac{1}{4}(1+\xi)(1+\eta), \\
N_{2}^{c}(\xi)=\frac{1}{4}(1+\xi)(1-\eta), & N_{4}^{c}(\xi)=\frac{1}{4}(1-\xi)(1+\eta) .
\end{array}
$$




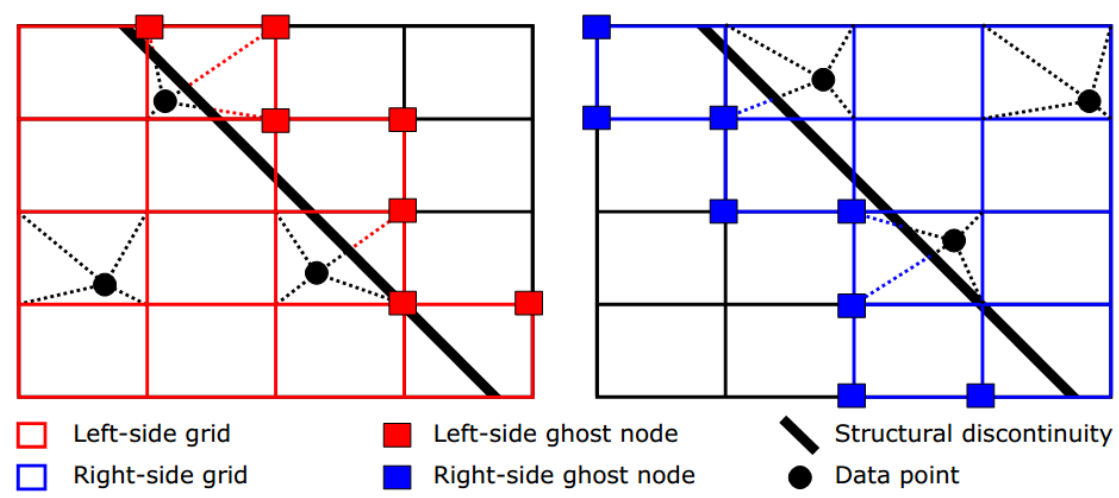

Figure 2: Illustration of the used ghost-cell technique by separating the evaluation on the left and on the right side of a discontinuity. Dotted segments represent the segment-discontinuity intersection tests for the use of ghost nodes.

\subsubsection{Handling the structural discontinuities}

Geological faults and unconformities are represented with a discontinuous jump in the implicit function $u(\boldsymbol{x})$. As a sum of continuous interpolation functions (eqn (1)), introducing a discontinuity in $u(\boldsymbol{x})$ calls for modifications either on the domain discretisation scheme, or on the interpolation functions. The presented method uses a ghost-cell technique [19], adding degrees of freedom on the grid cells crossed by discontinuities as illustrated in Figs 1 and 2. Points are added on either side of the discontinuities on top of already existing grid corners; in the following, the $N_{i}$ grid corners $\boldsymbol{x}_{l_{i}}$ are called interpolation nodes and the $N_{g}$ added points $\boldsymbol{x}_{l g}$ are called ghost nodes.

The implicit function is augmented to

$$
u(\boldsymbol{x})=\sum_{l_{i}=1}^{N_{i}} \Phi_{l_{i}}(\boldsymbol{x}) u_{l_{i}}+\sum_{l_{g}=N_{i}+1}^{N_{i}+N_{g}} \Phi_{l_{g}}(\boldsymbol{x}) u_{l_{g}}=\sum_{l=1}^{N} \Phi_{l}(\boldsymbol{x}) u_{l},
$$

with $N_{i}$ and $N_{g}$ the number of interpolation and ghost nodes respectively. When evaluating $u(\boldsymbol{x})$, if the position $\boldsymbol{x}$ belongs to a cell crossed by discontinuities, the cell corners used for interpolation (Section 2.1.2) are found following Fig. 2: if the segment between $\boldsymbol{x}$ and a corner (dotted line) intersects the discontinuity, then the node used for eqn (3) is the corresponding ghost node $\boldsymbol{x}_{l_{g}}$, otherwise it is the interpolation node $\boldsymbol{x}_{l_{i}}$. With this technique, the implicit function is continuously evaluated on either side of the discontinuity, regardless its geometry.

\subsection{Structural modelling problem}

The modelling problem (eqn (2)) is solved by least squares optimization. Equations related to the energy term are written on all the nodes, while equations related to the data term are written on the data points. 


\subsubsection{Data points equations}

Geological horizons are given by iso-values of the implicit function $u(\boldsymbol{x})$. An input data point is thus bound to its horizon $h$ by specifying its expected iso-value $\alpha_{h}$. A data equation of this type is written as

$$
\lambda_{k}\left(u\left(\boldsymbol{x}_{k}\right)-\alpha_{h}\right)=0, \forall \boldsymbol{x}_{k} \in h
$$

There is no a priori knowledge on the expected iso-values $\alpha_{h}$. The method approximates theses values during a pre-processing stage on input data which fixes the top and bottom horizons to a chosen value and then spatially interpolates the other horizons values linearly in between [20]. The data weights $\lambda_{k}$ are generally taken constant between all data but can vary depending on the reliability of the data (e.g., borehole data are generally more reliable than seismic data).

\subsubsection{Energy penalization}

The bending energy term is regularly discretised on $N_{i}$ cells $\Omega_{l_{i}}$ with the same volume $v$ and centred on each interpolation node $\boldsymbol{x}_{l_{i}}$. This discretisation thus represents the dual-grid of the interpolation grid. On each node, energy equations are written as

$$
\begin{aligned}
& \lambda_{\epsilon} \sqrt{v}\left(\frac{\partial^{2}}{\partial x x} u(\boldsymbol{x})\right)=0, \\
& \lambda_{\epsilon} \sqrt{v}\left(\frac{\partial^{2}}{\partial y y} u(\boldsymbol{x})\right)=0, \\
& \lambda_{\epsilon} \sqrt{2 v}\left(\frac{\partial^{2}}{\partial x y} u(\boldsymbol{x})\right)=0 .
\end{aligned}
$$

We chose to extend this discretisation to the ghost nodes: energy equations are also written on ghost nodes with the same volume of integration $v$.

The interpolation functions $\Phi_{l}(\boldsymbol{x})$ chosen in eqn (3) are only $C^{0}$ at the nodes $\boldsymbol{x}_{l}$. The second derivatives of eqn (6) are therefore approximated by finite difference as

$$
\begin{gathered}
\frac{\partial^{2}}{\partial x x} u\left(\boldsymbol{x}_{l}\right) \approx \frac{u\left(x_{l}+\Delta_{x}, y_{l}\right)-2 u\left(x_{l}, y_{l}\right)+u\left(x_{l}-\Delta_{x}, y_{l}\right)}{\Delta_{x}^{2}}, \\
\frac{\partial^{2}}{\partial y y} u\left(\boldsymbol{x}_{l}\right) \approx \frac{u\left(x_{l}, y_{l}+\Delta_{y}\right)-2 u\left(x_{l}, y_{l}\right)+u\left(x_{l}, y_{l}-\Delta_{y}\right)}{\Delta_{y}^{2}}, \\
\frac{\partial^{2}}{\partial x y} u\left(\boldsymbol{x}_{l}\right) \approx \\
\frac{u\left(x_{l}+\Delta_{x}, y_{l}+\Delta_{y}\right)-u\left(x_{l}+\Delta_{x}, y_{l}-\Delta_{y}\right)-u\left(x_{l}-\Delta_{x}, y_{l}+\Delta_{y}\right)+u\left(x_{l}-\Delta_{x}, y_{l}-\Delta_{y}\right)}{4 \Delta_{x} \Delta_{y}},
\end{gathered}
$$

where $\Delta_{x}$ and $\Delta_{y}$ are the grid spacing in the $x$ and $y$ axes respectively. As the interpolation functions $\Phi_{l}(\boldsymbol{x})$ have the Kronecker delta property (i.e., $\Phi_{l}\left(x_{k}\right)=\delta_{k l}, \forall(k, l) \in N ; u\left(\boldsymbol{x}_{l}\right)=$ $u_{l}, \forall l \in N$ ), eqns (7), (8), and (9) reduce to a linear combination of the coefficients $u_{l}$. We define the combination coefficients $\delta_{m}^{x x}\left(\boldsymbol{x}_{\boldsymbol{l}}\right), \delta_{m}^{y y}\left(\boldsymbol{x}_{l}\right)$ and $\delta_{m}^{x y}\left(\boldsymbol{x}_{l}\right)$ associated to the nodes $\boldsymbol{x}_{m}(m \in N)$ as illustrated on Fig. 3(a) for the three equations respectively. 
(a)

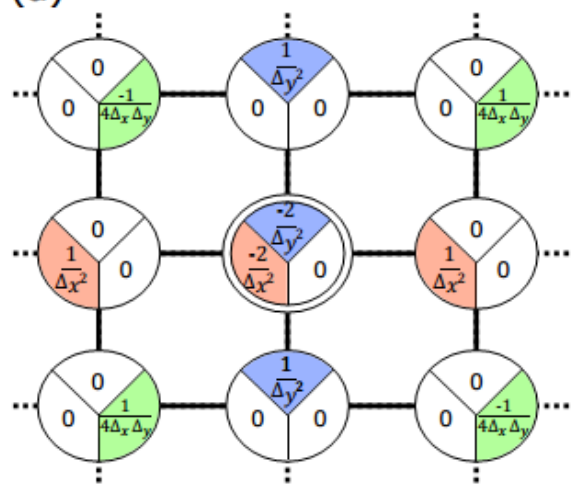

(c)
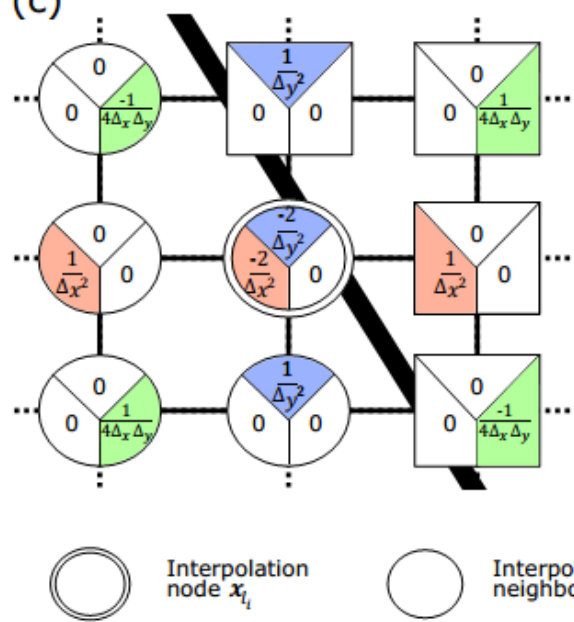

Interpolation node $x_{l_{i}}$

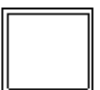

Ghost node $x_{l_{g}}$

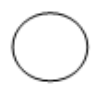

Interpolation neighbor $\boldsymbol{x}_{m}$

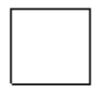
Ghost
neighbor $\boldsymbol{x}_{m_{g}}$ (b)

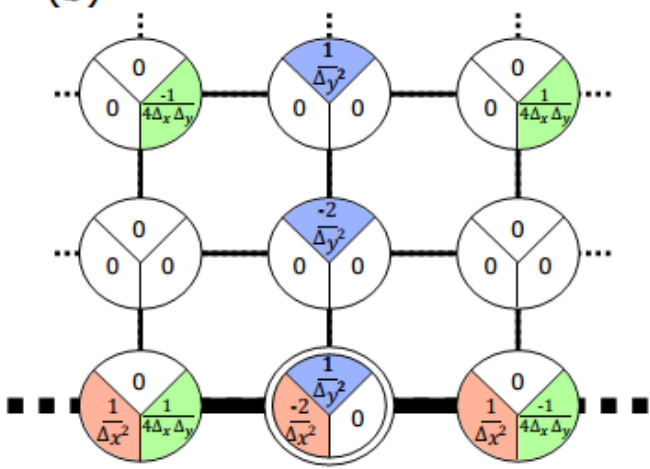

(d)

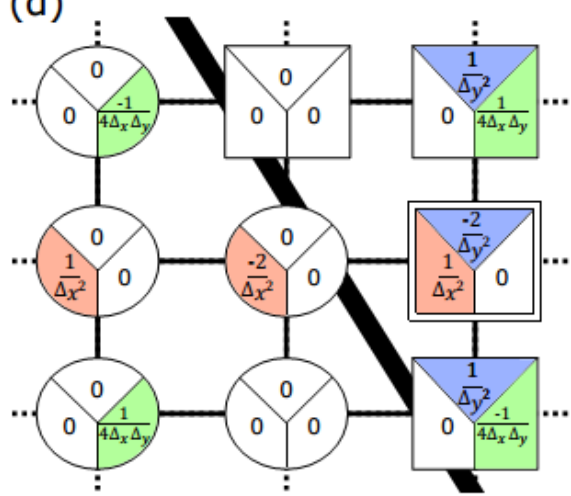

\begin{tabular}{|c|}
\hline$\delta_{m}^{x x}\left(\boldsymbol{x}_{l}\right)$ \\
\hline$\delta_{m}^{y y}\left(\boldsymbol{x}_{\mathrm{l}}\right)$ \\
\hline$\delta_{m}^{x y}\left(\boldsymbol{x}_{\mathrm{l}}\right)$ \\
\hline
\end{tabular}

Figure 3: Finite difference schemes for second derivatives with the related coefficients on different types of nodes. (a) An interpolation node; (b) An interpolation node on a border; (c) An interpolation node close to a discontinuity; and (d) A ghost node.

\subsubsection{System to solve}

Combining eqn (5), written on each data point $\boldsymbol{x}_{k}$, and eqns (7), (8), and (9), written on each node $\boldsymbol{x}_{l}$, we define the least squares system to solve, of size $(3 \mathrm{~N}+\mathrm{D}, \mathrm{N})$, as 


$$
\left[\begin{array}{ccc}
\lambda_{\epsilon} \sqrt{v} \delta_{1}^{x x}\left(x_{1}\right) & \cdots & \lambda_{\epsilon} \sqrt{v} \delta_{N}^{x x}\left(x_{1}\right) \\
\lambda_{\epsilon} \sqrt{v} \delta_{1}^{y y}\left(x_{1}\right) & \cdots & \lambda_{\epsilon} \sqrt{v} \delta_{N}^{y y}\left(x_{1}\right) \\
\lambda_{\epsilon} \sqrt{2 v} \delta_{1}^{x y}\left(x_{1}\right) & \cdots & \lambda_{\epsilon} \sqrt{2 v} \delta_{N}^{x y}\left(x_{1}\right) \\
\vdots & & \vdots \\
\lambda_{\epsilon} \sqrt{v} \delta_{1}^{x x}\left(x_{N}\right) & \cdots & \lambda_{\epsilon} \sqrt{v} \delta_{N}^{x x}\left(x_{N}\right) \\
\lambda_{\epsilon} \sqrt{v} \delta_{1}^{y y}\left(x_{N}\right) & \cdots & \lambda_{\epsilon} \sqrt{v} \delta_{N}^{y y}\left(x_{N}\right) \\
\lambda_{\epsilon} \sqrt{2 v} \delta_{1}^{x y}\left(x_{N}\right) & \cdots & \lambda_{\epsilon} \sqrt{2 v} \delta_{N}^{x y}\left(x_{N}\right) \\
\lambda_{1} \phi_{1}\left(x_{1}\right) & \cdots & \lambda_{1} \phi_{N}\left(x_{1}\right) \\
\vdots & & \vdots \\
\lambda_{D} \phi_{1}\left(x_{D}\right) & \cdots & \lambda_{D} \phi_{N}\left(x_{D}\right)
\end{array}\right] \cdot\left[\begin{array}{c}
u_{1} \\
\vdots \\
0 \\
0 \\
\vdots \\
0 \\
0 \\
0 \\
0 \\
\lambda_{N} \alpha_{1} \\
\vdots \\
\lambda_{D} \alpha_{D}
\end{array}\right]=\left[\begin{array}{c}
0 \\
\\
\\
0
\end{array}\right.
$$

After solving eqn (10), the implicit function $u(\boldsymbol{x})$ can be evaluated everywhere in the domain $\Omega$ using the coefficients $u_{l}$. If visualized on the grid of interpolation (i.e., the visualization points are the same as the corners of the grid), the implicit function values are given by the coefficients $u_{l_{i}}\left(l_{i}=\left\{1, \ldots, N_{i}\right\}\right)$. As our aim is to assess the quality of the interpolation also in the cells close to the discontinuities, the visualization grid is created finer than the interpolation grid and implicit values are evaluated using eqns (3) and (4). Fig. 1 was created using the proposed method on a $(15 \times 10)$ interpolation grid and a $(200 \times 200)$ visualization grid; energy weights $\left(\lambda_{\epsilon}\right)$ and data weights $\lambda_{k}(k=\{1, \ldots, D\})$ were set to 1 .

\section{HANDLING GHOST NODES IN STRUCTURAL GEOLOGICAL SETTINGS}

\subsection{Structural discontinuities with complex geometries}

\subsubsection{Fault tips}

Faults represent slip surfaces that can laterally vanish in space, where the displacement on each side of the fault becomes nil. The implicit function is supposed to be discontinuous on the fault and to become continuous at the fault tips. The chosen strategy to achieve the transition from an interpolation with ghost nodes to an interpolation without ghost nodes is illustrated at a fault tip in Fig. 4. As in Section 2.1.3, ghost nodes are added on either side of the fault and segment-discontinuity intersection tests are performed to define the nodes used in eqn (3) for the evaluation of the implicit function at a position $\boldsymbol{x}$.

\subsubsection{Intersections of discontinuities}

Several discontinuities (e.g., faults or erosions) can branch one onto another in space. Fig. 5 illustrates a simple case of two faults intersecting each other, defining three independent areas. The presented method creates as many ghost nodes as needed to interpolate on the cells close to the faults on each area. In this example, two ghost nodes are associated to each regular interpolation node, creating two additional coefficients $u_{l_{g}}$ for each corner. 


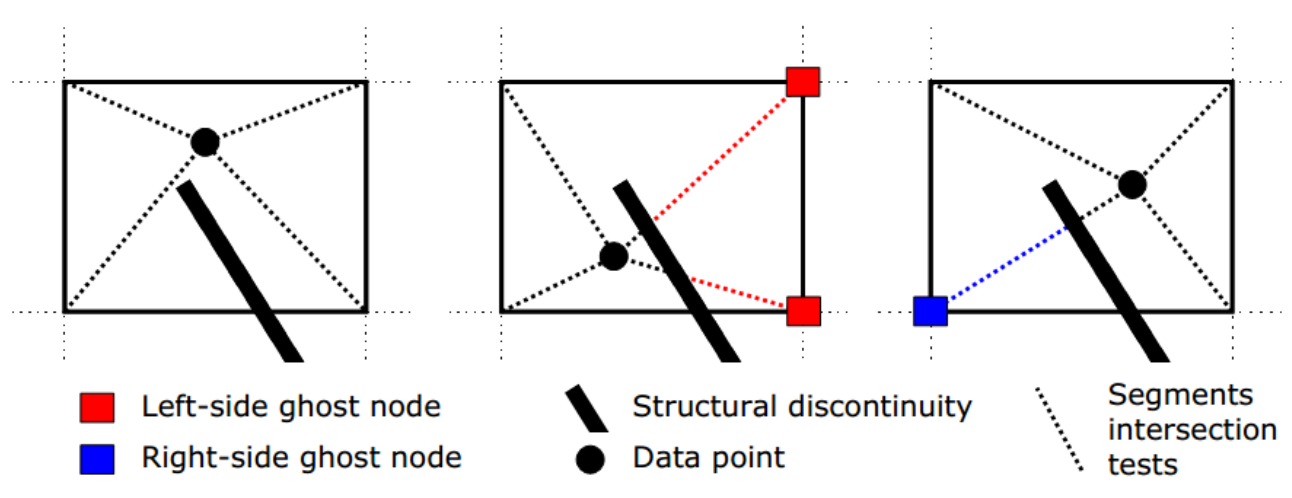

Figure 4: Illustration of the ghost-cell technique applied on a fault tip. A ghost node is used only if the segment between that node and a given data point intersects the discontinuity.

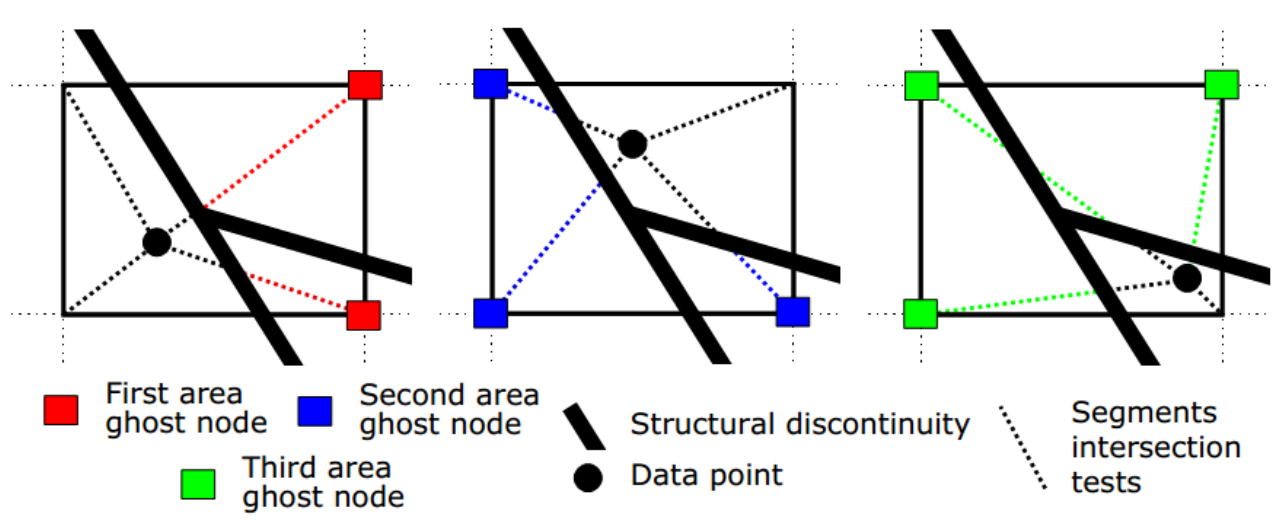

Figure 5: Illustration of the ghost-cell technique applied on several intersecting discontinuities. Ghost nodes are added for each area sealed by the discontinuities.

\subsection{Finite difference with ghost nodes}

The centred finite difference scheme presented in Fig. 3(a) can only apply to nodes far from the borders and the discontinuities. According to the discretisation of the energy term of eqn (2), the energy equations must be written on every interpolation node $\boldsymbol{x}_{l_{i}}$ and ghost node $\boldsymbol{x}_{l_{g}}$, which ensures homogeneous weights for all energy equations in the least squares system (10). Figs 3(b)-(d) illustrate the positions of the nodes $\boldsymbol{x}_{m}$ and their associated coefficients $\delta_{m}^{x x}\left(x_{l}\right), \delta_{m}^{y y}\left(x_{l}\right)$ and $\delta_{m}^{x y}\left(x_{l}\right)$ for three possible positions of a node $x_{l}$ with respect to the domain borders or the discontinuities. These configurations are often combined, on a border interpolation node close to a discontinuity for instance.

As in Section 2.1.3, the nodes $\boldsymbol{x}_{m}$ are found between interpolation and ghost nodes with segment-discontinuity intersection tests. 
(a)

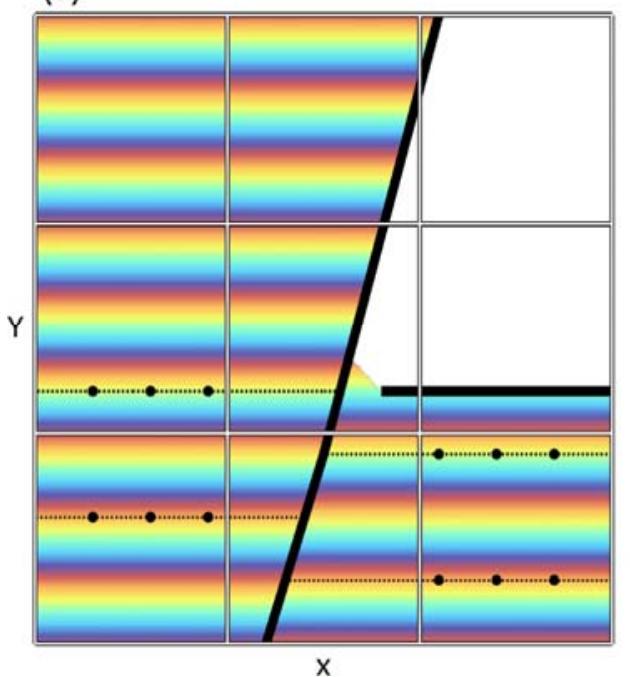

(b)

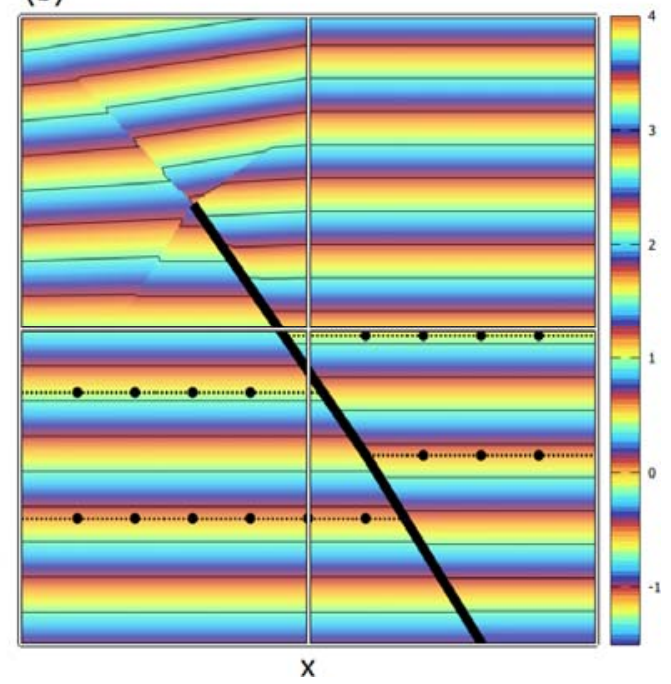

Figure 6: Resolution issues represented on two synthetic cross sections with faults and two parallel horizons. (a) Two disconnected faults treated as connected; (b) Rays of discontinuous jumps in the implicit function observed at the tip of a fault.

\subsection{Grid resolution issues}

The resolution of the grid can have a direct impact on the solution. In Fig. 6, the grid is chosen very coarse (i.e., (4x4) in Fig. 6(a) and (3x3) in Fig. 6(b)) to observe the influence on the resulting implicit function (observed on fine visualization grids of size (200x200) in both).

Fig. 6(a) shows how the method behaves when two faults are close to each other in the same cell but do not intersect: the connectivity between the two regions on the right side is not captured, the upper right area is left isolated without data, and hence has no solution. This is due to the way the nodes $\boldsymbol{x}_{m}$ are defined, using segment-discontinuity intersection tests (Sections 3.1.2 and 3.2). It shows that a resolution adapted to the scale of the geological structures being studied is expected with the proposed method.

Furthermore, the technique used at fault tips (Section 3.1.1) can generate discontinuous jumps in the implicit function within the containing cell (Fig. 6(b)). This is observed only because the visualization grid was taken way finer than the interpolation grid. This technique, based on segment-discontinuity intersection tests, is very close to the visibility criterion [21], which is known to generate the same artefacts [22]. Some other meshless techniques such as the diffraction and the transparency criteria [22], [23] could be tested.

\section{CONCLUSION AND PERSPECTIVES}

We proposed a grid based structural modelling method in two dimensions that handles discontinuities with a ghost-cell technique. The modelling problem is posed as a spatial regression of data points penalized by the bending energy approximated with finite difference schemes. The linear system to solve is thus sparse, and only the grid cells affected by discontinuities are stored in memory to handle the ghost nodes. 
The ghost-cell technique represents well complex structural geometries, with jumps in the implicit function only at discontinuities. However, the method is dependent on the resolution of the grid relatively to the spacing between the discontinuities and to the curvature of the underlying surfaces: a coarse grid may overlook finer structures, and it may generate finer artefacts in the implicit function close to fault tips. These issues could be respectively reduced with other tests than segment-discontinuity intersections to indicate the use of ghost nodes or using meshless optic criteria for instance [22], [23].

The method is presented in two dimensions, but the used formalism could be extended to three dimensions as well: with the adapted version of the bending energy, the corresponding finite difference schemes, and a definition of tests for discontinuities and ghost nodes based on three-dimensional objects.

\section{ACKNOWLEDGEMENTS}

This work was funded by Schlumberger, the Association Nationale Recherche Technologie, the Research for Integrative Numerical Geology consortium, and the Laboratory of Excellence Ressources 21 . These organisations are gratefully acknowledged.

\section{REFERENCES}

[1] Mallet, J.L., Three-dimensional graphic display of disconnected bodies. Mathematical Geology, 20(8), pp. 977-990, 1988. DOI: 10.1007/bf00892974.

[2] Lajaunie, C., Courrioux, G. \& Manuel, L., Foliation fields and 3D cartography in geology: Principles of a method based on potential interpolation. Mathematical Geology, 29(4), pp. 571-584, 1997. DOI: 10.1007/bf02775087.

[3] Chilès, J.P., Aug, C., Guillen, A. \& Lees, T., Modelling the Geometry of Geological Units and its Uncertainty in 3D From Structural Data: The Potential-Field Method. Orebody Modeling and Strategic Mine Planning-Spectrum 14, Jul. 22-24.

[4] Frank, T., Tertois, A.L., \& Mallet, J.L., 3d-reconstruction of complex geological interfaces from irregularly distributed and noisy point data. Computers \& Geosciences, 33(7), pp. 932-943, 2007. DOI: 10.1016/j.cageo.2006.11.014.

[5] Calcagno, P., Chilès, J.P., Courrioux, G, \& Guillen, A., Geological modelling from field data and geological knowledge. Physics of the Earth and Planetary Interiors, 171(1-4), pp. 147-157, 2008. DOI: 10.1016/j.pepi.2008.06.013.

[6] Caumon, G., Gray, G., Antoine, C. \& Titeux, M.O., Three-dimensional implicit stratigraphic model building from remote sensing data on tetrahedral meshes: theory and application to a regional model of La Popa Basin, NE Mexico. IEEE Transactions on Geoscience and Remote Sensing, 51(3), pp. 1613-1621, 2013.

DOI: $10.1109 /$ tgrs.2012.2207727.

[7] Hillier, M.J., Schetselaar, E.M., de Kemp, E.A. \& Perron, G., Three-Dimensional modelling of geological surfaces using generalized interpolation with radial basis functions. Mathematical Geosciences, 46(8), pp. 931-953, 2014.

DOI: $10.1007 / \mathrm{s} 11004-014-9540-3$.

[8] Souche, L., Iskenova, G., Lepage, F. \& Desmarest, D., Construction of structurally and stratigraphically consistent structural models using the volume-based modelling technology: Applications to an Australian dataset. International Petroleum Technology Conference, 2014.

[9] Mallet, J.L., Geomodeling, Oxford University Press, Inc., 2002.

[10] Mallet, J.L., Discrete Smooth Interpolation. Computer-aided Design, 24(4), pp. 178191, 1992. 
[11] Pellerin, J., Lévy, B., Caumon, G. \& Botella, A., Automatic surface remeshing of 3d structural models at specified resolution: A method based on Voronoi diagrams. Computers \& Geosciences, 62, pp. 103-116, 2014.

DOI: 10.1016/j.cageo.2013.09.008.

[12] Karimi-Fard, M. \& Durlofsky, L.J., A general gridding, discretization, and coarsening methodology for modeling flow in porous formations with discrete geological features. Advances in Water Resources, 96, pp. 354-372, 2016.

DOI: 10.1016/j.advwatres.2016.07.019.

[13] Renaudeau, J., Malvesin, E., Maerten, F. \& Caumon, G., Implicit structural modeling by minimization of the bending energy with local meshless techniques. In prep 2018.

[14] Irakarama, M., Laurent, G., Renaudeau, J. \& Caumon, G., Finite difference implicit modeling of geological structures. 80th EAGE Conference and Exhibition, Copenhagen: Denmark, 2018.

[15] Renaudeau, J., Malvesin, E., Maerten, F. \& Caumon, G., Implicit structural modeling with local meshless functions. 80th EAGE Conference and Exhibition, Copenhagen, Denmark, 2018.

[16] Dubrule, O., Comparing splines and kriging. Computers and Geosciences, 10(2-3), pp. 327-338, 1984. DOI: 10.1016/0098-3004(84)90030-x.

[17] Wahba, G., Spline models for observational data. Society for Industrial and Applied Mathematics, 59, 1990.

[18] Dhatt, G. \& Touzot, G., Une présentation de la méthode des éléments finis, Presses Université Laval, 1981.

[19] Fedkiw, RP, Aslam, T, Merriman, B. \& Osher, S., A non-oscillatory Eulerian approach to interfaces in multimaterial flows (the ghost fluid method). Journal of Computational Physics, 152(2), pp. 457-492, 1999. DOI: 10.1006/jcph.1999.6236.

[20] Collon, P., Steckiewicz-Laurent, W., Pellerin, J., Laurent, G., Caumon, G., Reichart, G. \& Vaute, L., 3D geomodelling combining implicit surfaces and Voronoi-based remeshing: A case study in the Lorraine coal basin (France). Computers \& Geosciences, 77, pp. 29-43, 2015. DOI: 10.1016/j.cageo.2015.01.009.

[21] Belytschko, T., Lu, Y.Y. \& Gu, L., Element-Free Galerkin methods. International Journal for Numerical Methods in Engineering, 37(2), pp. 229-256, 1994.

[22] Belytschko, T., Krongauz, Y., Fleming, M., Organ, D. \& Liu, W.K.S., Smoothing and accelerated computations in the Element Free Galerkin method. Journal of Computational and Applied Mathematics, 74(1-2), pp. 111-126, 1996. DOI: 10.1016/0377-0427(96)00020-9.

[23] Organ, D., Fleming, M., Terry, T. \& Belytschko, T., Continuous meshless approximations for nonconvex bodies by diffraction and transparency. Computational Mechanics, 18(3), pp. 225-235, 1996. DOI: 10.1007/s004660050142. 